\title{
Correlation Analysis of Elevation and the Relief Degree of Land Surface in Henan Province
}

\author{
Kaiguang Zhang*, Mingting Ba,Hongling Meng and Yanmin Sun \\ Zhengzhou Normal University, Zhengzhou 450044, China \\ ${ }^{*}$ Corresponding author
}

\begin{abstract}
Elevation and the relief degree of land surface are important factors in describing the regional topographic features. Studying their spatial relationship could further understand the spatial characteristics of the regional topographic distribution. Based on the $30 \mathrm{~m} \times 30 \mathrm{~m}$ digital elevation model, this paper uses raster algebra and spatial analysis method to calculate the relief degree of land surface and its correlation coefficient with elevation, analyzes their spatial distribution characteristics from the aspect of structure ratio, in order to explore their spatial relationship. The results indicate that the topography of Henan province is dominated by low and medium altitude elevation and low relief degree. the maximum values of elevation and the relief degrees in longitude lines and latitude lines show 4 ladders, their local differences are significant. the difference of amplitude is obvious in the transition zone around 1 mountain elevation, its variance is obviously higher than that in other mountain transition zones. The elevation of $300 \mathrm{~m}$ is the separatrix of the correlation coefficient change, on the left the correlation coefficient is directly proportional to elevation, and on the right side which is inversely proportional to elevation. There are clear correlations between the elevations and the relief degrees in 100$1300 \mathrm{~m}$ and $1500-1800 \mathrm{~m}$.
\end{abstract}

Keywords-digital elevation model; altitude elevation; the relief degree of land surface; correlation coefficient

\section{INTRODUCTION}

Regional topographic features restrict the redistribution of surface material and energy, directly affect the formation and development of soil and vegetation, determine the advantages and disadvantages of land use and land quality, and have an important impact on the formation of population distribution patterns[ $1,2,3]$. the Relief Degree of Land Surface(RDLS) refers to the contact degree of the altitude difference with the land surface in a given region, as a macro topographic factor in dividing of topography and geomorphology which is an important indicator to describing earth surface morphology. The study of the relief degree of land surface not only has mathematical significance, but also has the significance of soil erosion and geomorphology, has strong accuracy and practical application value in the evaluation of large-scale regional human-land relations. Elevation refers to the vertical distance of a point from the absolute datum plane, is a relative variable to describe the topographic features, which affects the formation and developing processes of soil and vegetation, as well as land use patterns to a large extent $[4,5]$.

In recent years, the researches about elevation and the relief degree of land surface impacts on the land use and regional economic development have mainly focused on the nature of land use and the spatial characteristics of population distribution, as well as their evolutionary process, have been obtained some valuable results[6-10]. In fact, although there are some correlations between elevation and relief degree in a region, there are certain differences in the distribution characteristics in different elevation ranges. The study of correlation between them in a region is helpful for deeply understanding of regional topography, to provide useful references for the effective use of natural resources.

This paper, based on the $30 \mathrm{~m} \times 30 \mathrm{~m}$ digital elevation model (DEM), utilizes raster algebra and spatial analysis method to calculate the relief degree of land surface and its correlation coefficient with elevation, and analyzes their spatial distribution characteristics from the aspect of structure ratio, explores their spatial relationship, in order to provide useful references for deeply understanding Henan topography.

\section{DATA AND RESEARCH METHODS}

\section{A. Research Area Overview and Data}

Henan Province $\left(31^{\circ} 23^{\prime} \mathrm{N}-36^{\circ} 22^{\prime} \mathrm{N}, 110^{\circ} 21^{\prime} \mathrm{E}-116^{\circ} 39^{\prime} \mathrm{E}\right)$ is located in the transition zone of China's terrain from the second to the third ladder, with the total area of 167 thousand $\mathrm{km}^{2}$. The three sides as the north, west and south are semicircular surrounded by Taihang, Funiu and Tabie mountains[9] The central and east regions are Huanghuaihai alluvial plain. $30 \mathrm{~m} \times 30 \mathrm{~m}$ DEM used in the study is provided by Computer Network Information Center (http://www. gscloud.cn).

\section{B. Research Methods}

The relief degree of land surface is an important index to describe the topographic features of a region, refers to the contact degree of the altitude difference with the land surface in a given region, defined as

$$
R D L S=A L T / 1000+[\operatorname{Max}(H)-\operatorname{Min}(H)] \times[1-P(A) / A] / 500
$$

where $A L T$ is the average elevation within the $\varepsilon$ neighborhood of the analysis unit, $\operatorname{Max}(H)-\operatorname{Min}(H)$ is the altitude difference in the neighborhood, $P(A)$ and A respectively are the surface area and the project area of the neighborhood. $500 \mathrm{~m}$ is a reference mountain height in China. As an independent value, the geographical significance of RDLS indicates that $R D L S \geq 1$ means the regional topography amplitude is in $\operatorname{int}(R D L S)$ 
reference mountains, $R D L S<1$ means the regional topography amplitude is below 1 reference mountain[5,6,9,11,12,].

Correlation coefficient is used to reflect the correlation degree between variables, by the sum of multiplied the dispersions of two variables. Suppose $\left(x_{i}, y_{i}\right), \quad i=1,2,3 \cdots n$ is the observations of two-dimensional random variable $(X, Y)$, and $\bar{x}, \bar{y}, D(X)$ and $D(Y)$ respectively are the mean and variance of $X$ and $Y$, the correlation coefficient of $X$ and $Y$ is defined as [14]

$$
\rho=\frac{\sum_{i=1}^{n}\left(x_{i}-\bar{x}\right)\left(\mathrm{y}_{i}-\bar{y}\right)}{\sqrt{D(X) D(Y)}}
$$

$\rho$ belongs to $[0,1]$, the greater $\rho$ means strong correlation, as $\rho=1$ means $X$ and $Y$ is linearly dependence, $\rho=0$ means $X$ and $Y$ mutual independence[13,14].

\section{CORRELATION ANALYSIS OF ELEVATION AND THE RELIEF DEgReE of LAND SURFACE IN HENAN PROVINCE}

\section{A. Distribution Characteristics of Elevation}

Figure 1a is the elevation distribution in Henan Province, Figure $1 \mathrm{~b}$ is the $45^{\circ}$ side view of the elevation distribution from the south focusing on the centroid, In order to show the elevation difference, the $\mathrm{z}$-value is magnified by 20 times. The elevation of the province (WGS84 ellipsoidal plane) is between $23.2 \mathrm{~m}$ and $2413.8 \mathrm{~m}$ with an average elevation of $403 \mathrm{~m}$. The province is dominated by medium and low elevations, the area of $180-500 \mathrm{~m}$ is about 128.4 thousand $\mathrm{km}^{2}$, occupies $90 \%$ of the total area, the area below $180 \mathrm{~m}$ accounts for $0.6 \%$ mainly distributed in the south and east part of the province, the region with the elevation between $500 \mathrm{~m}$ and $1500 \mathrm{~m}$ is the theme of the province mountain, with the area about 34.8 thousand $\mathrm{km}^{2}$, while the area above $1500 \mathrm{~m}$ is only 2 thousand $\mathrm{km}^{2}$ (Figure 1c and Figure 1d).
Figure1e is the curve of elevation statistics versus longitude, the maximum of elevation obviously shows 4 ladders from the west to the east. they respectively are the high altitude region located in the west of $112^{\circ} 24$ '36"E with the average maximum elevation about $1971 \mathrm{~m}$, and 38.4 thousand $\mathrm{km}^{2}$, the middle-high altitude region located between $112^{\circ} 24 ' 36^{\prime \prime} \mathrm{E}$ and $113^{\circ} 48^{\prime} 48^{\prime \prime} \mathrm{E}$ with an average maximum elevation of $1427 \mathrm{~m}$, and 43.4 thousand $\mathrm{km}^{2}$, the middle-low altitude region at $113^{\circ} 48^{\prime} 48^{\prime \prime} \mathrm{E}$ to $115^{\circ} 55^{\prime} 12^{\prime \prime} \mathrm{E}$ with the average maximum elevation of $849 \mathrm{~m}$, and 80.9 thousand $\mathrm{km}^{2}$, and the low altitude region in the east of $115^{\circ} 55^{\prime} 12^{\prime \prime} \mathrm{E}$ with the average maximum elevation of $244 \mathrm{~m}$, and 4.3 thousand $\mathrm{km}^{2}$ (Figure1c). The mean of the elevation decreases gradually with the increase of longitude, and the minimum value presents 2 ladders with the west-high east-low with $110^{\circ} 58^{\prime} 48^{\prime \prime} \mathrm{E}$ as the dividing line, and the western region average is $453 \mathrm{~m}$, accounting for $2.49 \%$ of the total area, the average value of the eastern region is $178.61 \mathrm{~m}$, accounting for $97.51 \%$ of the total area.

Figure $1 \mathrm{f}$ is the $45^{\circ}$ side view of the elevation distribution from the west focusing on the centroid(20 times $\mathrm{z}$ value), Figure $1 \mathrm{~g}$ is the curve of elevation statistics versus latitude. the maximum of elevation obviously shows 4 ladders from the south to the north. They respectively are the low altitude region located in the south of $33^{\circ} 14^{\prime} 24^{\prime \prime} \mathrm{N}$ with the average maximum elevation about $872 \mathrm{~m}$, the high altitude region located between $33^{\circ} 14^{\prime} 24^{\prime \prime} \mathrm{N}$ and $34^{\circ} 30^{\prime} 36^{\prime \prime} \mathrm{N}$ with an average maximum elevation of $1928 \mathrm{~m}$, the middle-low altitude region at $34^{\circ} 30^{\prime} 36^{\prime \prime} \mathrm{N}$ to $35^{\circ} 07^{\prime} 12^{\prime \prime} \mathrm{N}$ with the average maximum elevation of $1274 \mathrm{~m}$, and the middle-high altitude region in the north of $35^{\circ} 07^{\prime} 12^{\prime \prime} \mathrm{N}$ with the average maximum elevation of $1532 \mathrm{~m}$, their area respectively account for $31.36 \%, 41.28 \%$, $14.77 \%$ and $12.59 \%$ of the province area (Figure 1g). The mean value shows 2 parabola characteristics connecting at $33^{\circ}$ $39 ' 36 " \mathrm{~N}$. The minimum value is basically keeping at $132 \mathrm{~m}$ except for the north and south edges.

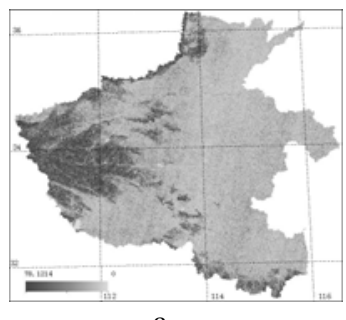

a

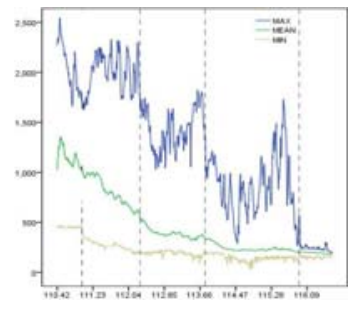

e

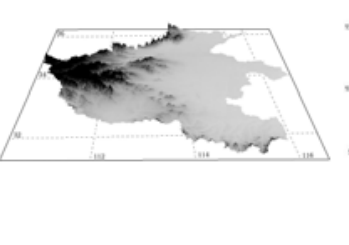

b

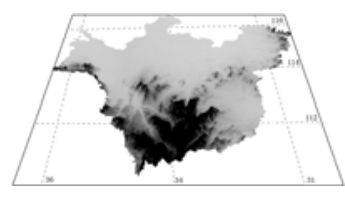

f
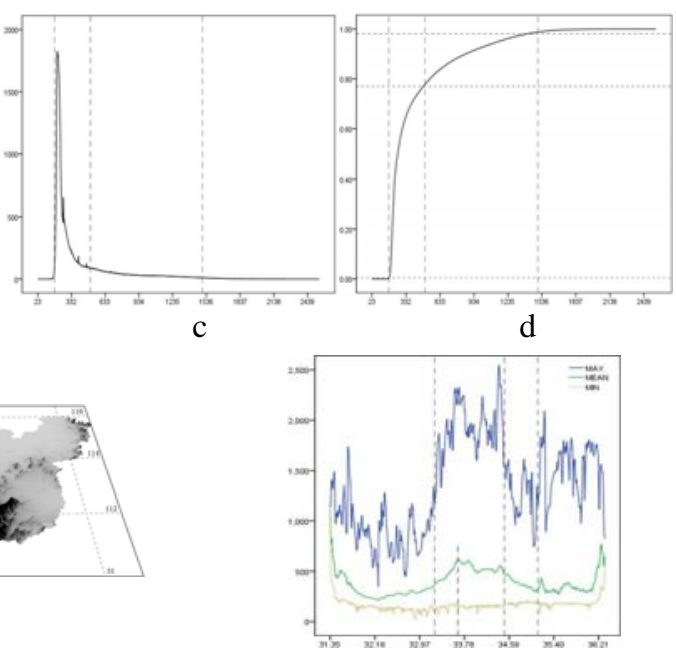

g

FIGURE I. 


\section{B. Distribution Characteristics of the Relief Degree of Land Surface}

Using Eq.1 and setting $\varepsilon=1 \mathrm{~km}$, to calculate the relief degree of land surface in Henan province, the results are showed in Figure 2, Figure 2a and Figure 2b respectively are $45^{\circ}$ side views of the relief degree of land surface from the south and west focusing on centroid, in order to show the variation, the relief degree of land surface is magnified by 10 thousand times. The relief degree of land surface in Henan Province is between 0.05-4.77. From the distribution density(Figure 2c) and area distribution (Figure 2d), it is dominated by low values, the area under 0.5 is $75.06 \%$ of the total area, with it increase, the area in the same dimension interval, the percentages decrease gradually, respectively are $0.5-2.5,12.02 \%, 6.59 \%, 3.61 \%, 1.79 \%$, as greater than 2.5 the area only accounts for $0.93 \%$.

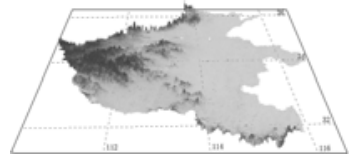

a

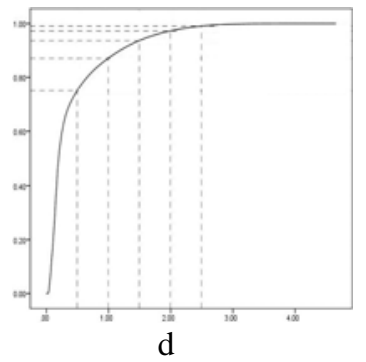

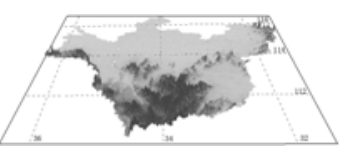

b

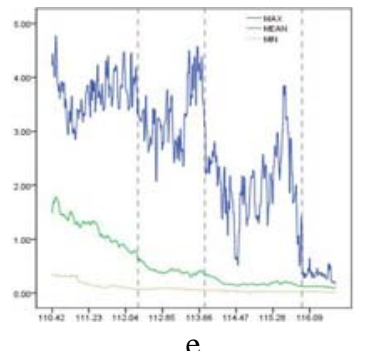

Figure 2e and $2 f$ respectively are the curve of the relief degree of land surface statistics versus longitude and altitude. Overall, there are great similarities between the change of elevation and the relief degree of land surface along longitude and latitude. On the longitude, the average maximum values in the 4 ladders respectively are 3.73, 3.33, 1.99 and 0.41 with areas about $36.90,43.80,81.70$ and 4.60 thousand $\mathrm{km}^{2}$. On the latitude, the average maximum values in the 4 ladders respectively are $1.95,3.60,2.56$ and 3.45 with areas about 52.4, 68.90, 26.10 and 19.60 thousand $\mathrm{km}^{2}$. Considering the characteristics of elevation distribution along longitude and latitude, there is a significant difference between the their changes around dividing lines, existing a significant correlation between them.

FIGURE II.

THE DISTRIBUTION CHARACTERISTICS OF THE RELIEF DEGREE OF LAND SURFACE IN HENAN PROVINCE

TABLE I. THE STATISTICS CHARACTERISTICS OF ELEVATION AND THE RELIEF DEGREE OF LAND SURFACE

\begin{tabular}{|c|c|c|c|c|c|c|c|c|c|c|c|c|c|}
\hline Class & 1 & 2 & 3 & 4 & 5 & 6 & 7 & 8 & 9 & 10 & 11 & 12 & 13 \\
\hline EM & 0.84 & 1.94 & 2.33 & 3.43 & 4.48 & 5.48 & 6.49 & 7.48 & 8.49 & 9.49 & 10.50 & 11.49 & 12.49 \\
\hline ESD & 16.71 & 22.27 & 26.37 & 28.59 & 29.00 & 28.72 & 28.99 & 28.74 & 28.81 & 28.94 & 28.92 & 28.74 & 28.85 \\
\hline HM & 0.35 & 0.13 & 0.16 & 0.34 & 0.55 & 0.70 & 0.87 & 1.02 & 1.18 & 1.34 & 1.47 & 1.59 & 1.72 \\
\hline HSD & 0.23 & 0.07 & 0.08 & 0.17 & 0.26 & 0.31 & 0.36 & 0.40 & 0.44 & 0.47 & 0.49 & 0.50 & 0.52 \\
\hline CC\% & 0.12 & 20.11 & 25.35 & 21.34 & 17.38 & 14.82 & 12.14 & 11.86 & 9.23 & 9.46 & 6.67 & 7.32 & 6.89 \\
\hline Class & 14 & 15 & 16 & 17 & 18 & 19 & 20 & 21 & 22 & 23 & 24 & 25 & \\
\hline EM & 13.48 & 14.48 & 15.47 & 16.46 & 17.45 & 18.46 & 19.46 & 20.45 & 21.43 & 22.42 & 23.41 & 24.42 & \\
\hline ESD & 28.81 & 28.75 & 28.65 & 28.61 & 28.65 & 28.70 & 28.59 & 28.39 & 27.99 & 28.10 & 28.67 & 28.10 & \\
\hline HM & 1.83 & 1.94 & 2.06 & 2.18 & 2.32 & 2.45 & 2.54 & 2.64 & 2.73 & 2.81 & 2.92 & 2.96 & \\
\hline HSD & 0.52 & 0.53 & 0.55 & 0.58 & 0.60 & 0.62 & 0.62 & 0.62 & 0.62 & 0.62 & 0.59 & 0.57 & \\
\hline CC $\%$ & 6.20 & 5.54 & 6.66 & 6.63 & 6.86 & 4.72 & 3.68 & 4.59 & 4.29 & 3.26 & 3.50 & 3.21 & \\
\hline
\end{tabular}




\section{Correlation Analysis of Elevation and the Relief Degree of Land Surface}

In order to study the relationship between elevation and the relief degree of land surface, according to the characteristics of elevation distribution in the province, this study divides the elevations into 25 classes with interval of $100 \mathrm{~m}$, and the $i^{\text {th }}$ class includes the elevation belongs to [100(i-1), 100i) (Figure 3a). According to Eq.2 by using the partition statistics and map algebra calculate the correlation coefficient of elevation and the relief degree of land surface, the results shown in Table 1.

The elevation means on the elevation series show the characteristics of linear increase, its linear regression equation is $E M=0.992 C L A S S-0.397\left(R^{2}=0.286\right)$, the linear regression equation after range standardization is $E M=0.042070$ CLASS -0.05246 , indicating that the elevation classification is relatively reasonable. However, as the average elevation increases, the standard deviation of elevation gradually increases, reaching a local maximum of 28.59 in the elevation $400 \mathrm{~m}$, and oscillating around 28.65 in the region above $400 \mathrm{~m}$, indicating that relief amplitude in the low altitude region is relatively small. the difference of relief amplitude is obvious in the transition zone around 1 mountain elevation, its variance is obviously higher than that in other mountain transition zones(Figure 3b).
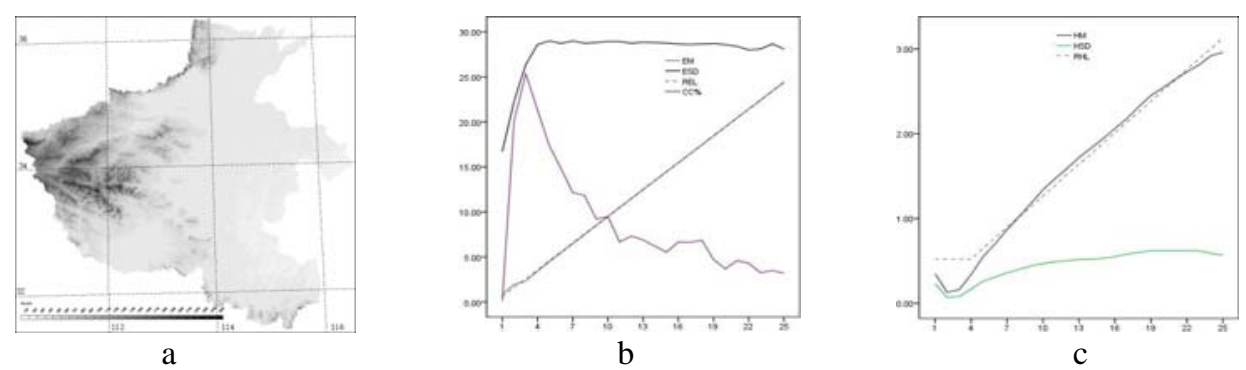

FIGURE III. THE DISTRIBUTION CHARACTERISTICS OF ELEVATION AND THE RELIEF IN HENAN PROVINCE

\section{CONCLUSION}

Based on the digital elevation model, the paper utilizes spatial analysis method and raster algebra to calculate the relief degree of land surface and its correlation coefficient with elevation , and analyzes the spatial distribution characteristics of elevation and the relief degree of land surface, studies their spatial correlations. The results show that:

(1) The topography of Henan province is dominated by low and medium altitude elevations, with the longitude increasing, the elevation maximum obviously shows 4 ladders, the elevation mean and elevation minimum gradually decreases. with the latitude increasing, the elevation maximum also shows 4 ladders, the elevation mean shows two connecting parabola characteristics.

(2) The topography of Henan province is dominated by low relief degree, its curves versus longitude and latitude is have great similarities with that of elevation, there is a significant difference between their changes around dividing lines, existing a significant correlation between them. The
With the elevation increases from 23 to 200m, the mean value of the relief degree of land surface decreases, and the mean value in $200-300 \mathrm{~m}$ basically remains at a relatively low level of 0.016 , above $300 \mathrm{~m}$, it shows some linear increasing characteristics, its linear regression equation after range standardization is $E M=0.041892$ CLASS $-0.10608\left(R^{2}=0.134\right)$, the relative gradient is obviously less than it of the average elevation. As the elevation increases from 23 to $200 \mathrm{~m}$, the standard deviation of the relief degree of land surface is gradually decreasing, it keeping at a lower level about 0.08 in 200-30m, and then increasing. Comparing with the elevation variation, its variations has a clear increasing trend. all of them show that with the elevation increasing, the relief amplitude in the same elevation zone becomes more obvious (Figure $3 \mathrm{~b}$ ).

With the elevation increases to $300 \mathrm{~m}$, the correlation coefficient between elevation and the relief degree of land surface increases, reaching a local maximum value of 0.2535 on $300 \mathrm{~m}$, and then gradually decreasing to 0.0321 on $2500 \mathrm{~m}$. The decreasing rate in $300-400 \mathrm{~m}$ is obviously higher than that in other intervals, the absolute change rate in $23-100 \mathrm{~m}$ is obviously highest. the correlation coefficients in the intervals of $100-1300 \mathrm{~m}$ and $1500-1800 \mathrm{~m}$ is higher than the relevant critical value of $6.25 \%[9]$, there is a clear correlation between elevation and the relief degree of land surface in these regions (Figure 3c).

difference of relief amplitude is obvious in the transition zone around 1 mountain elevation, its variance is obviously higher than that in other mountain transition zones.

(3) The elevation of $300 \mathrm{~m}$ is the separatrix of the correlation coefficient change, on the left with the increase of the elevation, the correlation coefficient between elevation and the relief degree increases, and on the right side which gradually decreases with the increase of elevation. There are clear correlations between the elevations and the relief degrees in $100-1300 \mathrm{~m}$ and $1500-1800 \mathrm{~m}$.

\section{REFERENCES}

[1] LI Jingjing, LU Zhemin, SHI Xiaoping, et al. Spatio temporal variations analysis for land use in Fen River Basin based on terrain gradient[J]. Transactions of the Chinese Society of Agricultural Engineering, 2016,32(7):230-236.

[2] Salazar Alvaro, Baldi Germán, Hirota Marina, et al. Land use and land cover change impacts on the regional climate of non-Amazonian South America: A review[J]. Global and Planetary Change, 2015, 128(5): 103119. 
[3] Fan Xingang, Ma Zhuguo, Yang Qing, et al. Land use/land cover changes and regional climate over the Loess Plateau during 2001-2009. Part II: Interrelationship from observations[J]. Climatic Change, 2014, 129(3/4): 441-455.

[4] Cai Daoming,Li Bai, Xu Wensheng,et.al.. Relief degree of land surface in Hubei province studied based on ASTERGDEM dada and its correlation with population density and economic development[J]. Bulletion of Soil and Water Conservation, 201737(5):231-240.

[5] Ran Hong. Extaction of relief amplitude in Chongqing city and its application in the assessment of water and soil loss[J]. Anhui Agri.Sci.Bull, 2017,23(09):92-94.

[6] Lu Zhixiang, Yang Yonggang, Zou Songbing,et al. A study of the land use change and its hydrologic response in the upper reaches of the Fen River[J]. Journal of Glaciology and Geocryology, 2014, 36(1): 192-199. (in Chinese with English abstract)

[7] Liu Jiyuan, KuangWenhui, Zhang Zengxiang, et al. Spatiotemporal characteristics, patterns and causes of land use changes in China since the late 1980s[J]. Acta Geographica Sinica, 2016,61(1):3-14.

[8] Pachauri A K, Gupta P V, Chander R. Landslide zoning in a part of the Garhwal Himalayas. Environmental Geology, 1998, 36(3-4): 25-334.

[9] K.G. Zhang, H.L. Meng,M.T. Ba, et al.. Correlation analysis of the population and land use distribution with the relief degree of land surface in Henan province. 2017 2nd international conference on applied mathematics, simulation and modelling (AMSM2017), DEStech Transactions on Engineering and Technology Research,94-100.

[10] Y. Liu, W. Deng, X.X. Song, et al.. Population Density Correction Method in Mountain Areas Based on Relief Degree of Land Surface:A Case Study in the Upper Minjiang River Basin. Scientia Geographica Sinica, 2015,36(4):464-469.

[11] T.T.Chen, L.Peng, S.Q.Liu,et al, Relationships of relief degree of topography with population and economy in Hengduan mountain area based on GIS, Journal of University of Chinese Academy of Sciences,2016, 33( 4) : 505-512.

[12] T.Z. Shu, X.H. Wang. Research for the correlation between relief amplitude and regional soil erosion based on 3S technolog. Research of Soil and Water Conservation,2017,4(24):127-132.

[13] H.X.Gao. Applied multivariate statistical analysis. Peking University Press, 2015.

[14] R.Z.Guo. Spatial analysis, Higher Education Press,Beijing,2011. 